\title{
Water-drinking Test and Pharmacologic Mydriasis as Provocative Tests in Primary Angle Closure Suspects
}

\author{
Reza Razeghinejad ${ }^{1,2}$, MD; M. Hossein Nowroozzadeh ${ }^{2}$, MD \\ ${ }^{1}$ Glaucoma Service, Wills Eye Hospital, Philadelphia, PA, USA \\ ${ }^{2}$ Poostchi Ophthalmology Research Center, Shiraz University of Medical Sciences, Shiraz, Iran \\ ORCID: \\ Reza Razeghinejad: https://orcid.org/0000-0001-7961-8425 \\ M. Hossein Nowroozzadeh: https://orcid.org/0000-0002-7412-1900
}

\section{Abstract}

Purpose: To compare the water-drinking test (WDT) and pharmacologic mydriasis as provocative tests in patients with primary angle closure suspect (PACS).

Methods: This observational non-randomized comparative study evaluated changes in intraocular pressure (IOP) in 21 patients with PACS who underwent pharmacologic mydriasis and compared it with IOP changes in 26 patients given the WDT. Ocular biometric and anterior chamber parameters were also assessed. Tests were repeated on the same patient two weeks after performing laser peripheral iridotomy (LPI).

Results: The mean age \pm standard deviation was $60 \pm 7$ and $57 \pm 9$ years in the mydriasis and WDT groups, respectively $(P=0.201)$. Before $\mathrm{LPI}$, both provocative tests were associated with a significant increase in IOP (mydriasis: $15.1 \pm 3.1$ to $16.6 \pm 3.5 \mathrm{mmHg}, P=0.025$; WDT: $16.2 \pm 2.8$ to $18.5 \pm 3.3 \mathrm{mmHg}, P<0.001$ ). However, the IOP changes were not statistically different between groups $(P=0.102)$. After LPI, only the WDT group showed a continued significant IOP elevation after the test (mydriasis: $16.4 \pm 3.3$ to $16.7 \pm 3.5 \mathrm{mmHg}, P=$ 0.569; WDT: $14.9 \pm 3.0$ to $17.8 \pm 4.1 \mathrm{mmHg}, P<0.001)$. The post-test IOP change was significantly greater in the WDT than in the mydriasis group ( 3.0 versus $0.3 \mathrm{mmHg}$, respectively; $P=0.002$ ). Step-wise multiple regression analysis verified the type of provocative test as the only independent factor affecting the post-test IOP change after LPI (regression coefficient: $2.664 ; P=0.002$ ).

Conclusion: Pharmacologic mydriasis and the WDT had similar IOP elevation before LPI, but after LPI, IOP elevation was much greater in the WDT group.

Keywords: Intraocular Pressure; Pharmacologic Mydriasis; Primary Angle Closure Suspect; Water-drinking Test

J Ophthalmic Vis Res 2019; 14 (3): 267-274

\section{Correspondence to:}

M. Hossein Nowroozzadeh, MD. Poostchi Ophthalmology Research Center, Poostchi Clinic, Zand St., Shiraz 71349, Iran.

E-mail: nowroozzadeh@hotmail.com

Received: 04-02-2018 Accepted: 05-09-2018

\section{Access this article online}

Website:

https://knepublishing.com/index.php/JOVR

DOI:

10.18502/jovr.v14i3.4782

\section{INTRODUCTION}

Primary angle closure glaucoma (PACG) is believed to be one of the leading causes of irreversible blindness with approximately 20 million people experiencing PACG worldwide. ${ }^{[1-3]}$

This is an open access journal, and articles are distributed under the terms of the Creative Commons Attribution-NonCommercial-ShareAlike 4.0 License, which allows others to remix, tweak, and build upon the work non-commercially, as long as appropriate credit is given and the new creations are licensed under the identical terms. 
The spectrum of angle closure disease includes a narrow angle with $180^{\circ}$ or more of iridotrabecular apposition (primary angle closure suspect [PACS]), a narrow angle with increased intraocular pressure (IOP) or peripheral anterior synechiae (primary angle closure, [PAC]), and PAC with optic neuropathy or PACG. ${ }^{[4]}$ It has been reported that approximately $10 \%$ of PACS patients eventually develop glaucoma. ${ }^{[2]}$ While there is some evidence that a prophylactic laser peripheral iridotomy (LPI) significantly reduces the risk of angle closure glaucoma in the contralateral eye of an individual with angle closure glaucoma, there is much confusion regarding the need for a prophylactic LPI for patients with asymptomatic narrow angles detected through gonioscopy. Although LPI is an effective preventive therapy, we must consider the large number of candidates involved and the burden and cost of treating ten-folds for each eye that receives an actual benefit. Therefore, identifying PACS eyes that would benefit from LPI through appropriate imaging or provocative tests is desirable. Various provocative tests including the dark-room provocative test and pharmacologic mydriasis have been used to identify narrow angle eyes at risk of angle closure. ${ }^{[5]}$ Pharmacologic or dark-induced mydriasis leads to a relative pupillary block and increased iridotrabecular contact. The prone position in the dark room test may induce forward movement of the lens and enhance the effect of the relative pupillary block. ${ }^{[6]}$ However, this provocative test has a low sensitivity and positive predictive value in detecting eyes susceptible to angle closure glaucoma. ${ }^{[7]}$ Therefore, we need to identify more appropriate provocative tests to determine PACS patients with the greatest risk of PACG and learn more about the mechanism of angle closure formation.

The water-drinking test (WDT) is used as a provocative test for assessing the outflow facility of aqueous humor in patients with primary openangle glaucoma. Choroidal expansion is a suggested mechanism of IOP elevation in WDT. ${ }^{[8,9]}$ Choroidal expansion has also been suggested as one of the mechanisms involved in PACG development. ${ }^{[10]}$ The expansion of the choroid after WDT could push the iris-lens diaphragm forward and result in a decrease in anterior chamber volume. ${ }^{[8,11]}$ Therefore, this test has the potential to be a provocative test in PACS. This study aimed to evaluate the changes in the IOP, ocular biometric, and anterior chamber parameters in PACS patients who underwent WDT or pharmacologic mydriasis, before and after LPI.

\section{METHODS}

This observational non-randomized comparative study enrolled 47 consecutive patients with PACS who were referred to the glaucoma clinic of a tertiary eye care center. The first 21 referred patients received the pharmacologic mydriasis test and the next 26 patients underwent WDT. All procedures performed in this study were in accordance with the 1964 Helsinki Declaration and its later amendments. The study was approved by the local Ethics Committee and informed consent was obtained from all patients. The patients underwent a comprehensive ophthalmologic examination including slit lamp biomicroscopy, Goldmann applanation tonometry (Haag Streit AG, Bern, Switzerland), indentation gonioscopy using a Sussman 4-mirror gonio lens (Volk Optical Inc., Mentor, OH, USA), and stereoscopic assessment of the optic disc with a 90 diopter lens (Volk Optical Inc., Mentor, $\mathrm{OH}$, USA). Subjects were classified as PACS if they presented $\geq 180^{\circ}$ of iridotrabecular contact, without peripheral anterior synechiae in indentation gonioscopy, glaucomatous optic neuropathy, or IOP $>22 \mathrm{mmHg} .{ }^{[12]}$ The exclusion criteria consisted of a history of ocular trauma, prior intraocular or refractive surgery, any intraocular disorder except cataract, secondary angle closure glaucoma, evidence of active keratitis or cornel pathology precluding gonioscopy and fundus examination, and the use of miotics or anticholinergics, pregnancy, hypertension, cardiac or kidney diseases, history of urinary retention, or inability to cooperate with any of the study measurements.

\section{Water-drinking Test (WDT)}

To perform the WDT, the patients refrained from food and fluid intake for three hours preceding the test. Patients were instructed to drink one liter of bottled water within five minutes. All WDTs were performed between 12 to $2 \mathrm{pm}$. The original WDT developed for primary open angle glaucoma detection and the majority of recent studies assessing the effect of WDT on IOP fluctuation used one liter of water for this purpose. ${ }^{[13-16]}$ Therefore, we opted to use one liter instead of the $10-15 \mathrm{~mL} / \mathrm{kg}$ 
reported by other studies. The measurements were obtained at baseline and at 30 minutes after drinking the water. ${ }^{[8]}$ The patients then underwent LPI using a commercial ophthalmic Nd: YAG laser system (Nidek YC-1800, Nidek Inc., Japan) one week after the WDT. The laser parameters were as follows: one pulse with a power of 4-6 mJ performed approximately 30 minutes after applying one drop of $2 \%$ pilocarpine eye drops. ${ }^{[17]}$ Two weeks after LPI, all measurements were repeated before and after 30 minutes of WDT.

\section{Mydriasis Test}

The measurements were obtained before and after the induction of pharmacologic mydriasis. In order to eliminate any possible effects of cycloplegic agents on the ciliary muscles and lens position, mydriasis was achieved with phenylephrine $5 \%$ drops applied every five minutes for a total of two applications. LPI was performed the week following the mydriasis test. All measurements were repeated before and after mydriasis, two weeks after LPI.

\section{Measurements}

The main outcome measure was IOP change after the tests. An increase in IOP of $\geq 6 \mathrm{mmHg}$ from baseline was considered a positive result on both provocative tests. ${ }^{[18]}$ Secondary outcomes were refraction ocular biometric parameters obtained using Lenstar LS 900 (Haag-Streit AG, Koeniz, Switzerland) and Pentacam HR (Oculus, Wetzlar, Germany) optical biometers. The following parameters were collected from the Lenstar biometer: mean keratometry $(\mathrm{Km})$, keratometric astigmatism (Ka), central corneal thickness (CCT), axial length $(A L)$, and lens thickness (LT). The Pentacam HR was used to obtain $\mathrm{Km}$, central anterior chamber depth (ACD; from the endothelium to the anterior lens surface), anterior chamber volume, and inferior anterior chamber angle. The following factors were calculated from the Lenstar recordings: lens-axial length factor $[\mathrm{LAF}=(\mathrm{LT} / \mathrm{AL}) \times 10]$; lens position $(\mathrm{LP}$ $=A C D+0.5 \times L T)$; and relative lens position $[R L P$ $=(\mathrm{LP} / \mathrm{AL}) \times 10]$. Each instrument was calibrated at the beginning of the study, and at regular intervals afterward (as per manufacturer's recommendations). All measurements were performed by the same experienced investigator using the criteria provided by the manufacturer of each device.

\section{Statistical Analysis}

IBM SPSS Statistics software version 21 (SPSS Inc., Chicago, IL) and MedCalc version 12.2.1 (MedCalc Software, Mariakerke, Belgium) were used for statistical analyses. Data from one eye of each patient, chosen at random, were included in the analysis. Descriptive data were presented as mean ( \pm standard deviation). The normality of data was assessed using the Kolmogorov-Smirnov test. The paired-samples $T$-test (or its nonparametric counterpart, the Wilcoxon-signed Rank test) was used to compare data before and after each test. The independent-sample $T$-test (or Mann-Whitney $U$ test) was used to compare post-test changes between different groups. Linear regression analysis was used to evaluate the effects of different parameters on the test results. A $P$-value of less than 0.05 was considered statistically significant.

\section{RESULTS}

Data from 21 PACS patients in the pharmacologic mydriasis group were compared with 26 patients in the WDT group. Table 1 summarizes the baseline characteristics of patients in each group. Before LPI, both provocative tests were associated with a significant IOP elevation (Table 2). IOP elevation in the WDT group was almost twice of the mydriasis group (2.4 versus 1.3); however, the difference was not statistically significant. Following LPI, the WDT group had a significant increase in IOP after the test, while the pharmacologic mydriasis group did not [Table 2; Figure 1]. The post-LPI WDT IOP-change was significantly greater in the WDT than the mydriasis group (3.0 versus $0.3 \mathrm{mmHg}$, respectively; $P=0.002$ ). Before $\mathrm{LPI}$, two (7.6\%) patients in the WDT and one (4.7\%) in the mydriasis group had positive test results $(\geq 6 \mathrm{mmHg}$ increase in IOP), but after LPI, seven (26.9\%) patients in the WDT group and none of the patients in the mydriasis group had positive test results $(P=0.01$; Chi-Square test).

There were no significant differences in biometric parameters for both tests before and after LPI (data not shown). Because the two studied groups were not similar in terms of sex and refraction, we conducted a linear regression analysis to assess 
Table 1. Baseline characteristics of patients suspected of primary angle closure that underwent provocative testing with either the pharmacologic mydriasis or water-drinking test

\begin{tabular}{lccc}
\hline Provocative test & Pharmacologic mydriasis & Water-drinking test & P-value \\
\hline Number & 21 & 26 & $57 \pm 9$ \\
Age, years & $60 \pm 7$ & $22 / 4$ & 0.201 \\
Sex, M/F & $4 / 17$ & 0.001 \\
Spherical equivalent of refraction, D & $1.3 \pm 1.2$ & $22.4 \pm 0.8$ & 0.036 \\
Axial length, mm & $22.5 \pm 0.6$ & $2.24 \pm 0.24$ & 0.731 \\
Anterior chamber depth, $\mathrm{mm}$ & $2.20 \pm 0.21$ & $94.3 \pm 17.6$ & 0.579 \\
Anterior chamber volume, $\mu \mathrm{m}^{3}$ & $88.2 \pm 13.7$ & $28.2 \pm 5.5$ & 0.200 \\
Anterior chamber angle, degrees & $25.6 \pm 7.0$ & $535 \pm 28$ & 0.167 \\
Central corneal thickness, $\mu \mathrm{m}$ & $527 \pm 35$ & $44.7 \pm 1.7$ & 0.426 \\
Mean keratometry, D & $44.6 \pm 1.2$ & 0.736 \\
\hline
\end{tabular}

$\mathrm{D}$, diopter; F, female; $\mathrm{M}$, male; mm, millimeter; $\mu \mathrm{m}$, micrometer

Table 2. Comparison of pharmacologic mydriasis and water-drinking tests as provocative tests in patients suspected of primary angle closure before and after laser peripheral iridotomy

IOP $(\mathrm{mmHg})$ before laser peripheral iridotomy

\begin{tabular}{|c|c|c|c|c|}
\hline & Before test & After test & P-value & Changes* \\
\hline Pharmacologic mydriasis & $15.1 \pm 3.1$ & $16.6 \pm 3.5$ & 0.025 & $1.3 \pm 2.2$ \\
\hline Water-drinking Test & $16.2 \pm 2.8$ & $18.5 \pm 3.3$ & $<0.001$ & $2.4 \pm 2.1$ \\
\hline$P$-value & & & & 0.102 \\
\hline \multicolumn{5}{|c|}{ IOP $(\mathrm{mmHg})$ after laser peripheral iridotomy } \\
\hline Pharmacologic mydriasis & $16.4 \pm 3.3$ & $16.7 \pm 3.5$ & 0.569 & $0.3 \pm 2.5$ \\
\hline Water-drinking test & $14.9 \pm 3.0$ & $17.8 \pm 4.1$ & $<0.001$ & $3.0 \pm 2.9$ \\
\hline$P$-value & & & & 0.002 \\
\hline
\end{tabular}

*Defined as: Post-test IOP - Pre-test IOP

IOP, intraocular pressure

the effects of different factors on the post-test IOP changes after LPI (Table 3). In simple regression analysis, sex, and the type of the provocative test appeared to be associated with the observed posttest IOP changes after LPI $(P<0.1)$. Figure 2 shows the effects of sex on IOP changes after provocative tests were performed before and after LPI. Sex had no significant association with IOP changes either before or after LPI (Mann-Whitney U test). In line with this finding, step-wise multiple regression analysis showed that the sex had no independent effect, and the type of the provocative test was the only independent factor affecting the post-test IOP changes after LPI (regression coefficient: 2.664; $P$ $=0.002$ ).

\section{DISCUSSION}

Our results showed that the amount of IOP elevation after pharmacologic mydriasis was less than WDT-IOP elevation before and after LPI. There was no statistically significant difference between the pharmacologic mydriasis and WDT groups in terms of IOP elevation before LPI, but after LPI, the increase in IOP was statistically significantly greater in the WDT group. After LPI, the IOP changes were lower in the mydriasis group compared with 
Table 3. Regression analysis of possible factors affecting IOP change after provocative tests of water-drinking test and pharmacologic mydriasis in patients with primary angle closure suspect after laser peripheral iridotomy

\begin{tabular}{lcc}
\hline Factors & Simple Linear Regression & $\begin{array}{c}\text { Multiple Regression } \\
\text { P-value }\end{array}$ \\
\hline Age & P-value & 0.332 \\
Sex & $0.094^{*}$ & 2.664 \\
Test (WDT vs. pharmacologic mydriasis) & $0.002^{*}$ \\
Baseline IOP & 0.225 \\
SE Refraction & 0.359 \\
Anterior chamber depth & 0.406 \\
Anterior chamber volume & 0.301 \\
Anterior chamber angle & 0.278 \\
Axial Length & 0.565 \\
Central corneal thickness & 0.814 \\
Mean keratometry & 0.118 \\
\hline
\end{tabular}

*Factors with $P$-value less than 0.1 were entered into a multiple stepwise regression model

IOP, intraocular pressure; SE, spherical equivalent; WDT, water-drinking test

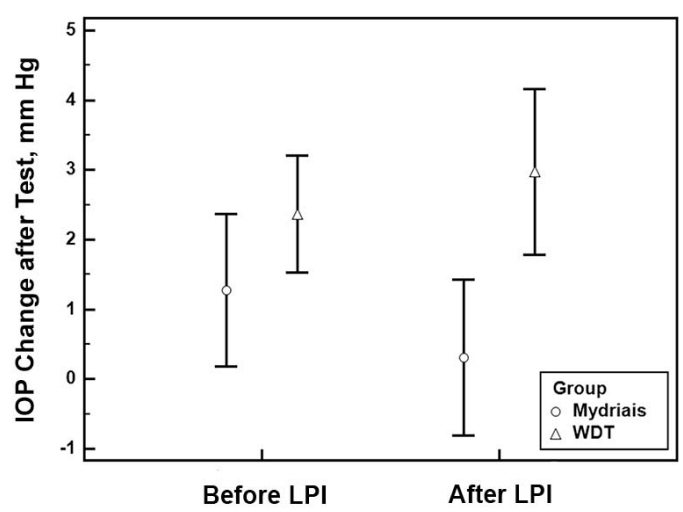

Figure 1. Comparison of intraocular pressure (IOP) changes after the water-drinking test (WDT) and pharmacologic mydriasis in primary angle closure suspect patients before and after laser peripheral iridotomy (LPI).

before LPI measurements, while the IOP changes were greater in the WDT group in measurements obtained after LPI than before LPI.

After LPI, the IOP changes in the mydriasis group were lower than before LPI (1.3 versus $0.3 \mathrm{mmHg}$ ), while the differences were greater in the WDT group (2.4 versus $3.00 \mathrm{mmHg}$ ). Moreover, there was a greater number of patients with positive WDT test results after LPI compared to before LPI.
The lower post-LPI IOP change in the mydriasis group could be attributed to the resolution of the pupillary block component of IOP elevation. A higher change in WDT-IOP after LPI compared to before LPI has been reported previously in PACS patients. ${ }^{[19]}$ The WDT-IOP elevation mechanisms may not be dependent on the pupillary block, but it is directly associated with the outflow capability of aqueous humor. In a previous study, in 16 out of 18 eyes subjected to argon laser iridotomy, there was evidence of decreased trabecular outflow after LPI in tonography. ${ }^{[20]}$ The decreased outflow may be explained by the possibility of damage from pigment release and inflammation after LPI to the already somehow impaired trabecular meshwork function due to the long-term apposition to the iris.

The WDT is a stress test that was initially developed to screen for open-angle glaucoma; however, its diagnostic capabilities showed that the WDT lacked the sensitivity and specificity needed to be a reliable screening test. Currently, the WDT is used to evaluate the aqueous outflow facility and the efficacy of surgical and medical glaucoma management, in addition to predicting the diurnal IOP peak. ${ }^{[21,22]}$ Sympathetic stimulation via yet-tobe-determined mechanisms has been suggested to be responsible for the WDT-IOP elevation. ${ }^{[23]}$ Increased iris thickness, owing to sympathetic 

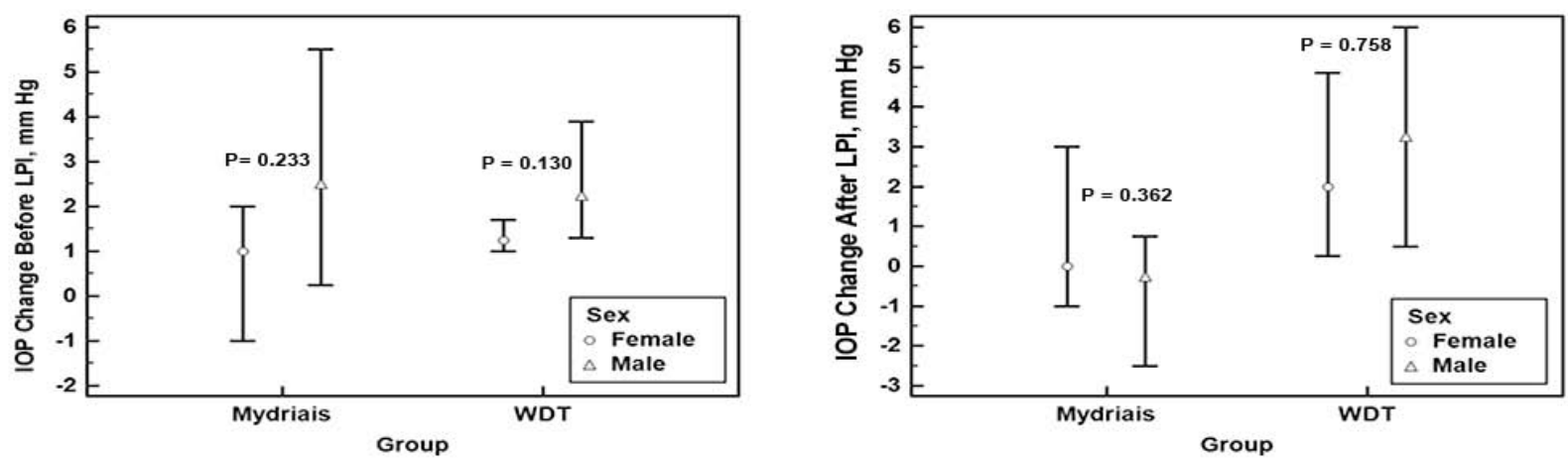

Figure 2. Effect of sex on intraocular pressure (IOP) changes after the water-drinking test (WDT) and pharmacologic mydriasis in primary angle closure suspect patients before and after laser peripheral iridotomy (LPI).

stimulation of the iris dilator muscle and its resulting circumferential folding, may prompt angle closure in patients with narrow angle glaucoma. Moreover, a significant increase in choroidal thickness and IOP elevation and a decrease in anterior chamber depth after WDT have been observed in angle closure but not in open angle eyes. ${ }^{[8]}$ The lack of any changes in the ocular biometric parameters in our patients of both groups is in line with other studies on the angle closure patients. ${ }^{[24-27]}$ In a group of fellow eyes of 21 patients with acute primary angle closure and in 17 patients with PACG, the ACD did not change following WDT. ${ }^{[26]}$ The study by Poon et al on PACG and primary openangle glaucoma revealed no association between WDT-IOP changes and anterior chamber depth and axial length. ${ }^{[25]}$ In a study on patients with PACS, PAC, and PACG, the positive dark room and mydriasis tests were defined as an increase in IOP of $6 \mathrm{mmHg}$. There was no difference in the anterior chamber depth between positive and negative testers (1.95 versus $1.89 \mathrm{mmHg}, P=0.3$ ) with the dark room test, but it was shallower in the patients with a positive mydriasis test $(1.77$ versus $1.96 \mathrm{mmHg}, P=0.010$ ). This difference could have resulted from the limited number of patients in each subgroup because the suggested mechanisms underlying both tests are similar. ${ }^{[27]}$ In a study on PACS patients, no changes in ocular biometric or anterior chamber parameters including iridocorneal angle after peripheral iridotomy and/or pharmacologic mydriasis except for increments in anterior chamber volume were observed. ${ }^{[24]}$
The mechanisms involved in WDT-IOP elevation are still unknown and may be different in open and closed angle eyes. The resolution of the pupillary block after LPI and the lower IOP elevation observed following pharmacologic mydriasis and the greater IOP elevation with WDT may exclude the possibility of the pupillary block as the main mechanism of WDT-IOP elevation in patients with closed angle eyes. Long-term follow-up of patients for IOP elevation may clarify the role of WDT in this group of patients.

Although it has been suggested that LPI be performed for narrow angles that fulfill the criteria of PACS, the decision of which subjects require prophylactic LPI remains highly subjective. It has been estimated that approximately one out of ten patients with anatomically narrow angles develop angle closure, ${ }^{[28]}$ therefore, the option of treating all patients with narrow angles would result in a large majority undergoing unnecessary procedures and a waste of resources. There is a great demand to develop a test that could identify PACS likely to progress to PAC. It has been suggested that angle closure may often be caused by other mechanisms, which would not be resolved by an iridotomy, such as peripheral iris crowding, a plateau iris or lens-related angle closure, and other unknown mechanisms. ${ }^{[29]}$ Interestingly, it has been reported that the extent of PAS did not correlate with the rise in IOP seen on provocative tests such as the dark room test, pharmacologic mydriasis, and the Valsalva test in patients with PAC. ${ }^{[18]}$ Parameters identifying eyes that continue to develop angle closure after an iridotomy would lead to better 
management of PAC. Although LPI is claimed to prevent PACS progression to PAC or PACG, it is not guaranteed, as some patients progress despite successful LPI. IOP elevation after WDT, but not after pharmacologic mydriasis, probably involves mechanisms other than pupillary block.

Limitations of our study include the relatively small sample size; thus our results require validation in future studies involving more patients. In addition, inherent variability of IOP measurements because of diurnal changes and device repeatability issues might affect the results. We limited IOP measurements to the same time frame on each day to minimize the confounding effects. Another limitation is the lack of angle grading information. Because the purpose of this study did not include the evaluation of angles before and after the LPI, we did not include the gonioscopy grading. However, patients in both groups had similar anterior chamber depth, anterior chamber angle width, anterior chamber volume, and axial length, which could indicate a lack of notable differences in angle configuration between the two groups. Performing pharmacologic mydriasis before LPI may be regarded as an ethical limitation of the current study as it may induce an acute angle closure attack. None of the eyes in our study developed an acute attack during the tests and the IOPs prior to and following the LPI were very similar. Diagnostic mydriasis appears to be relatively safe in patients with narrow angle. The risk of acute angle closure attack after pupillary dilation has been reported to be $0.03-0.3 \% .{ }^{[30,31]}$ Moreover, in a study in the UK, $81 \%$ of ophthalmologists doing provocative testing for PACS patients used pupillary dilation and only $15 \%$ used the dark room provocative test. ${ }^{[32]}$ Nevertheless, we explained the risks of the test to all patients. Finally, there was a difference in the sex ratio between both groups, but in the multiple regression analysis, the only determinant factor affecting the IOP changes of the provocative tests were the tests. Another limitation could be the lack of long-term follow-up of the patients and the correlation of the final glaucoma status with the initial test results; however, conducting such a study would be difficult to execute.

In conclusion, both pharmacologic mydriasis and WDT resulted in IOP elevation before LPI, but after LPI the IOP elevation was greater in the WDT group, which could be explained by the resolution of the pupillary block and consequently the lower IOP elevation in the pharmacologic mydriasis group. The mechanism of IOP elevation of WDT does not seem to be related to the biometric changes observed in PACS patients. Pharmacologic mydriasis and WDT may involve different mechanisms for IOP elevation, which may have the potential to advance our understanding about the mechanism of angle-closure glaucoma.

\section{Financial Support and Sponsorship}

Nil.

\section{Conflicts of Interest}

There are no conflicts of interest.

\section{REFERENCES}

1. Quigley HA, Broman AT. The number of people with glaucoma worldwide in 2010 and 2020. Br J Ophthalmol 2006;90:262-267.

2. Foster PJ, Oen FT, Machin D, Ng TP, Devereux JG, Johnson GJ, et al. The prevalence of glaucoma in Chinese residents of Singapore: A cross-sectional population survey of the Tanjong Pagar district. Arch Ophthalmol 2000;118:1105-1111.

3. Quigley HA. Glaucoma. Lancet 2011;377:1367-1377.

4. Sihota R. Classification of primary angle closure disease. Curr Opin Ophthalmol 2011;22:87-95.

5. Tornquist R. Dark-room test on eyes with a shallow anterior chamber. Acta Ophthalmol (Copenh) 1958;36:664-671.

6. Kondo T, Miyazawa D, Unigame K, Kurimoto Y. Ultrasound biomicroscopic findings in humans with shallow anterior chamber and increased intraocular pressure after the prone provocation test. Am J Ophthalmol 1997;124:632640.

7. Wilensky JT, Kaufman PL, Frohlichstein D, Gieser DK, Kass MA, Ritch R, et al. Follow-up of angle-closure glaucoma suspects. Am J Ophthalmol 1993;115:338-346.

8. Arora KS, Jefferys JL, Maul EA, Quigley HA. Choroidal thickness change after water drinking is greater in angle closure than in open angle eyes. Invest Ophthalmol Vis Sci 2012;53:6393-6402.

9. De Moraes CG, Reis AS, Cavalcante AF, Sano ME, Susanna $\mathrm{R}$ Jr. Choroidal expansion during the water drinking test. Graefes Arch Clin Exp Ophthalmol 2009;247:385-389.

10. Quigley HA. What's the choroid got to do with angle closure? Arch Ophthalmol 2009;127:693-694.

11. Quigley HA. Angle-closure glaucoma-simpler answers to complex mechanisms: LXVI Edward Jackson Memorial Lecture. Am J Ophthalmol 2009;148:657-669.

12. Razeghinejad MR, Myers JS. Contemporary approach to the diagnosis and management of primary angle-closure disease. Surv Ophthalmol 2018;63:754-768.

13. Leydhecker W. The water-drinking test. $\mathrm{Br} J$ Ophthalmol 1950;34:457-479. 
14. Leighton DA, Phillips $\mathrm{Cl}$, Gibbs AC. Provocative outflow test. Combining water drinking and homatropine. $\mathrm{Br} J$ Ophthalmol 1970;54:19-26.

15. Kumar RS, de Guzman MH, Ong PY, Goldberg I. Does peak intraocular pressure measured by water drinking test reflect peak circadian levels? A pilot study. Clin Exp Ophthalmol 2008;36:312-315.

16. Vasconcelos-Moraes CG, Susanna R Jr. Correlation between the water drinking test and modified diurnal tension curve in untreated glaucomatous eyes. Clinics (Sao Paulo) 2008;63:433-436.

17. Esmaeili A, Barazandeh B, Ahmadi S, Haghi A, Ahmadi Hosseini SM, Abolbashari F. Assessment of the anterior chamber parameters after laser iridotomy in primary angle close suspect using Pentacam and gonioscopy. Int $J$ Ophthalmol 2013;6:680-684.

18. Sihota R, Rishi K, Srinivasan G, Gupta V, Dada T, Singh K. Functional evaluation of an iridotomy in primary angle closure eyes. Graefes Arch Clin Exp Ophthalmol 2016;254:1141-1149.

19. Waisbourd M, Savant SV, Sun Y, Martinez P, Myers JS. Water-drinking test in primary angle-closure suspect before and after laser peripheral iridotomy. Clin Exp Ophthalmol 2016;44:89-94.

20. Karmon G, Vender T, Savir H. Evaluation of laser iridectomy in angle-closure glaucoma: provocative tests. $\mathrm{Br} \mathrm{J}$ Ophthalmol 1982;66:471-473.

21. Danesh-Meyer HV, Papchenko T, Tan YW, Gamble GD. Medically controlled glaucoma patients show greater increase in intraocular pressure than surgically controlled patients with the water drinking test. Ophthalmology 2008;115:1566-1570.

22. Medeiros FA, Pinheiro A, Moura FC, Leal BC, Susanna R Jr. Intraocular pressure fluctuations in medical versus surgically treated glaucomatous patients. J Ocul Pharmacol Ther 2002;18:489-498.
23. Venugopal N. Water drinking test and angle closure glaucoma. Indian J Ophthalmol 2015;63:172.

24. Razeghinejad MR, Lashkarizadeh $\mathrm{H}$, Nowroozzadeh $\mathrm{MH}$, Yazdanmehr M. Changes in ocular biometry and anterior chamber parameters after pharmacologic mydriasis and peripheral iridotomy in primary angle closure suspects. $J$ Optom 2016;9:189-195.

25. Poon YC, Teng MC, Lin PW, Tsai JC, Lai IC. Intraocular pressure fluctuation after water drinking test in primary angle-closure glaucoma and primary open-angle glaucoma. Indian J Ophthalmol 2016;64:919-923.

26. Nongpiur ME, Foo VH, de Leon JM, Baskaran M, Tun $\mathrm{TA}$, Husain R, et al. Evaluation of choroidal thickness, intraocular pressure, and serum osmolality after the water drinking test in eyes with primary angle closure. Invest Ophthalmol Vis Sci 2015;56:2135-2143.

27. Yamada R, Hirose F, Matsuki T, Kameda T, Kurimoto Y. Comparison of mydriatic provocative and dark room prone provocative tests for anterior chamber angle configuration. J Glaucoma 2016;25:482-486.

28. Wang $\mathrm{N}, \mathrm{Wu} \mathrm{H}$, Fan Z. Primary angle closure glaucoma in Chinese and Western populations. Chin Med J (Engl) 2002;115:1706-1715.

29. Yeung BY, Ng PW, Chiu TY, Tsang CW, Li FC, Chi CC, et al. Prevalence and mechanism of appositional angle closure in acute primary angle closure after iridotomy. Clin Exp Ophthalmol 2005;33:478-482.

30. Wolfs RC, Grobbee DE, Hofman A, de Jong PT. Risk of acute angle-closure glaucoma after diagnostic mydriasis in nonselected subjects: the Rotterdam Study. Invest Ophthalmol Vis Sci 1997;38:2683-2687.

31. Patel KH, Javitt JC, Tielsch JM, Street DA, Katz J, Quigley HA, et al. Incidence of acute angle-closure glaucoma after pharmacologic mydriasis. Am J Ophthalmol 1995;120:709-717.

32. Sheth HG, Goel R, Jain S. UK national survey of prophylactic YAG iridotomy. Eye (Lond) 2005;19:981-984. 